\title{
Hydrogen bond dynamics and vibrational spectral diffusion in aqueous solution of acetone: A first principles molecular dynamics study ${ }^{\#}$
}

\author{
BHABANI S MALLIK ${ }^{\mathrm{a}, \mathrm{b}}$ and AMALENDU CHANDRA ${ }^{\mathrm{a}, *}$ \\ ${ }^{a}$ Department of Chemistry, Indian Institute of Technology Kanpur, Kanpur 208016, India \\ ${ }^{b}$ Present address: Department of Chemistry, Indian Institute of Technology Hyderabad, Yeddumailaram \\ 502205, India \\ e-mail: amalen@iitk.ac.in
}

\begin{abstract}
We present an ab initio molecular dynamics study of vibrational spectral diffusion and hydrogen bond dynamics in aqueous solution of acetone at room temperature. It is found that the frequencies of OD bonds in the acetone hydration shell have a higher stretch frequency than those in the bulk water. Also, on average, the frequencies of hydration shell OD modes are found to increase with increase in the acetone-water hydrogen bond distance. The vibrational spectral diffusion of the hydration shell water molecules reveals three time scales: A short-time relaxation ( $\sim 80 \mathrm{fs}$ ) corresponding to the dynamics of intact acetone-water hydrogen bonds, a slower relaxation $(\sim 1.3 \mathrm{ps})$ corresponding to the lifetime of acetone-water hydrogen bonds and another longer time constant $(\sim 12 \mathrm{ps})$ corresponding to the escape dynamics of water from the solute hydration shell. The present first principles results are compared with those of available experiments and classical simulations.
\end{abstract}

Keywords. Ab initio molecular dynamics; spectral diffusion; acetone-water; hydrogen bond dynamics.

\section{Introduction}

This paper is concerned with calculations of vibrational spectral diffusion and hydrogen bond dynamics in an aqueous solution of acetone from first principles simulations. When acetone is dissolved in water, hydrogen bonds can be formed between the carbonyl oxygen of acetone and hydrogen of water molecules in its first hydration shell and the strength and dynamics of these hydrogen bonds can greatly influence the energetics, dynamics and vibrational characteristics of both water and acetone molecules in the solutions. Also, the water molecules that are hydrogen bonded to the carbonyl group can behave differently from those near the hydrophobic methyl group of the acetone solute or from those of the bulk water. The goal of the present study is to investigate such differences in the equilibrium and dynamical behaviour of water molecules in the acetone hydration shell as compared to those outside the hydration shell with a special focus on their hydrogen bond kinetics and frequency fluctuations. We note that such a study is extremely relevant not only in solution chemistry but also in biological chemistry where water molecules can frequently come in direct contact with such type of polar groups.

\footnotetext{
\# Dedicated to Prof. N Sathyamurthy on his 60th birthday

*For correspondence
}

For water-acetone systems, in addition to the hydrophilic hydrogen bonded solvation of the carbonyl group, there is also the hydrophobic solvation of the methyl groups of acetone by water molecules and these different solvation characteristics are believed to give rise to very different water-acetone interactions as compared to the interactions in the respective neat liquids. The interest in studying these mixtures can also be gauged by the many experimental ${ }^{1-10}$ and theoreti$\mathrm{cal}^{11-15}$ studies that have already been devoted to look at various macroscopic and molecular properties of wateracetone systems. For example, Chapados and coworkers ${ }^{10}$ carried out Fourier transform infrared attenuated total reflectance spectroscopic studies of acetone-water mixtures. These authors found that the $\mathrm{OH}$ stretch band is blue shifted as acetone is added to water. Recently, Bakker and coworkers ${ }^{16}$ looked at the dynamical properties of water molecules that are directly hydrogenbonded to acetone molecules at high concentration and enclosed in a fluid organic matrix by using femtosecond mid-IR pump-probe laser spectroscopy. These authors also found weaker acetone-water hydrogen bonds compared to those in bulk water, however, the dynamics of water-acetone hydrogen bonds was found to have a slower time scale because of confinement. Very recently, Gupta et al. ${ }^{17}$ carried out classical molecular dynamics simulations of water-acetone mixtures of varying composition and found a faster dynamics of water-acetone hydrogen bonds than those 
between water molecules. So, the acetone-water hydrogen bonds were calculated to be weaker than waterwater bonds in agreement with IR experiments. ${ }^{10}$ There have also been a few studies on acetone-water system through $a b$ initio molecular dynamics ${ }^{18-21}$ and combined quantum-classical (QM/MM) ${ }^{22-24}$ methods, but these studies have not looked at the fluctuations of acetone-water hydrogen bonds or the vibrational spectral diffusion which originate from such hydrogen bond fluctuations. We address these latter issues in the present work by means of ab initio molecular dynamics without using any empirical interaction potentials.

In the present work, we have carried out a first principles theoretical study of vibrational spectral diffusion and hydrogen bond dynamics in dilute aqueous solution of an acetone molecule by employing the method of $a b$ initio molecular dynamics ${ }^{25,26}$ and wavelet analysis. ${ }^{27-29} \mathrm{In}$ addition to looking at the hydration shell structure and frequency-structure correlations, we have also looked at the dynamics of hydration shell water around an acetone molecule, especially for those molecules which are hydrogen bonded to the carbonyl oxygen. We first looked at the distribution of hydration shell OD frequencies as compared to that of bulk water and also the influence of hydrogen bonds on the OD stretch frequencies and how they correlate with the hydrogen bond distance. Subsequently, we calculated the hydrogen bond and residence dynamics of hydration shell molecules by means of population time correlation function approach and the dynamics of vibrational spectral diffusion through frequency time correlation calculations. The observed dynamics of the spectral diffusion of hydration shell water molecules are analysed in terms of the dynamics of the intact acetone-water hydrogen bonds, lifetime of acetonewater hydrogen bonds and also the residence time of water molecules in the acetone hydration shell.

\section{Details of simulations and frequency calculations}

The $a b$ initio molecular dynamics simulations have been carried out by employing the Car-Parrinello method $^{25,26}$ and the CPMD code. ${ }^{30}$ The present system contains a single acetone molecule dissolved in a solvent of $31 \mathrm{D}_{2} \mathrm{O}$, which was kept in cubic box of length 10.17 ̊. Periodic boundary conditions were applied in all the three dimensions and the electronic structure of the extended systems was represented by the density functional theory within a plane wave basis. The core electrons were treated via the atomic pseudopotentials of Troullier-Martins ${ }^{31}$ and the plane wave expansion of the orbitals was truncated at a kinetic energy of $80 \mathrm{Ry}$. We employed the so-called BLYP ${ }^{32}$ functional in the present study. This functional has been used in earlier ab initio molecular dynamics simulations of acetonewater systems ${ }^{18,19}$ and also for a host of other hydrogen bonded systems such as liquid and supercritical water, ${ }^{33-41}$ aqueous ionic solutions, ${ }^{42-50}$ methanol, ${ }^{51-53}$ ammonia $^{54,55}$ and also mixed liquids such as watermethanol mixtures. ${ }^{56}$ Previous studies ${ }^{18,19}$ have shown that BLYP yields good results for solvatochromic shift of acetone in water when compared with the corresponding experimental results, hence it reliably captures the solute-solvent interactions in these aqueous solutions of acetone. On the dynamical side, this functional, like many other functionals within generalized gradient approximation (GGA), predicts a somewhat slower translational and rotational diffusion of aqueous systems ${ }^{37-39}$ which, in part, could be the fact that the GGA functionals do not describe the dispersive interactions satisfactorily. The BLYP functional has also been used for simulating chemical reactions in aqueous media. ${ }^{57}$ For proton transfer processes in aqueous solutions, it has been shown recently that BLYP performs reliably while some of the other functionals produced results which were inconsistent with experiments. ${ }^{58,59}$

In the present simulations, a fictitious mass of $\mu=$ 800 a.u. was assigned to the electronic degrees of freedom and the coupled equations of motion describing the system dynamics was integrated by using a time step of 5 a.u. All hydrogen atoms were assigned the mass of deuterium to reduce the influence of quantum effects on the dynamical properties. Also, our choice of deuterium ensures that electronic adiabaticity and energy conservation are maintained throughout the simulations for the chosen values of the fictitious electronic mass parameter and time step. We note that the choice of a proper value of the fictitious electronic mass parameter is an important issue in carrying out Car-Parrinello molecular dynamics in the correct manner. The proper value of the electronic mass paremeter to be used in a simulation depends on various factors such as nuclear mass, time step, temperature, etc. The value of 800 a.u. has been found to be acceptable for the present systems as no significant drift of the electronic kinetic energy was observed during the entire simulations. Hence, we do not expect any significant effects of the electronic drag on nuclear motion due to the fictitious electronic mass parameter used in the present study. The initial configuration of water molecules and acetone were generated by carrying out a classical molecular dynamics simulation. For water molecules, we have used the SPC/E interaction potential ${ }^{60}$ and for acetone, an OPLS all atom model ${ }^{61}$ is adopted. Then, during $a b$ initio 
molecular dynamics simulation, we equilibrated the system for $8 \mathrm{ps}$ in canonical ensemble at $300 \mathrm{~K}$ and, thereafter, we continued the run in NVE ensemble for another $60 \mathrm{ps}$ for averaging of structural and dynamical properties. We note that this run period of $60 \mathrm{ps}$, although seem quite short compared to typical lengths of classical simulations, is rather long in the context of ab initio molecular dynamics. To our knowledge, all of the existing $a b$ initio simulations of aqueous acetone solutions ${ }^{18-21}$ involved run lengths which were significantly shorter than than that of the present simulations.

It is known that the vibrational frequency of an OD bond in the solutions fluctuates due to fluctuations in its interactions with the surrounding molecules. A quantitative calculation of the time dependent vibrational frequencies of OD bonds can be carried out through a time series analysis of the $a b$ initio molecular dynamics trajectories using the wavelet method. ${ }^{27}$ This method has already been discussed in our previous studies of pure water ${ }^{39}$ and, therefore, we refer the reader to these references for methodological details of the time dependent frequency calculations of all the OD modes of the solution along the simulation trajectory.

\section{Vibrational frequencies of water in the vicinity of acetone}

In figure 1a, we have shown the frequency distributions of OD bonds which are hydrogen bonded to acetone and also of those which are in the bulk water. For this figure and also for figure 2 shown later, we have used the MATLAB package to smooth the raw simulation data using the loess method with a span of about $5-10$ percent. ${ }^{62}$ The existence of a hydrogen bond between the $\mathrm{D}$ (of OD) and the carbonyl oxygen of acetone $\left(\mathrm{O}_{a}\right)$ is found out by using a simple geometric criterion that the $\mathrm{D} \cdot \mathrm{O}_{a}$ distance should be less than $2.4 \AA$. On the other hand, a water molecule is taken to be in the solvation shell of $\mathrm{O}_{a}$ when their $\mathrm{O} \cdot \mathrm{O}_{a}$ distance is less than $3.10 \AA$. Note that these distances correspond to the first minimum of the intermolecular $\mathrm{D} \cdot \cdot \mathrm{O}_{a}$ and $\mathrm{O} \cdot \cdot \mathrm{O}_{a}$ radial distribution functions (figure 1b). The corresponding cut-off distances for water-water hydrogen bonds are taken to be 2.45 and $3.35 \AA$, respectively (figures not shown). Integration of the acetone-water radial distribution functions up to their first minima give coordination and hydrogen bond numbers of 2.05 and 1.8 for the carbonyl oxygen. In figure 1a, we observe a blue shift in the frequencies of hydration shell OD bonds as compared to those of the bulk OD modes. The average frequencies of the hydration shell $\left(\bar{\omega}_{\text {hyd }}\right)$ and bulk $\left(\bar{\omega}_{b}\right)$
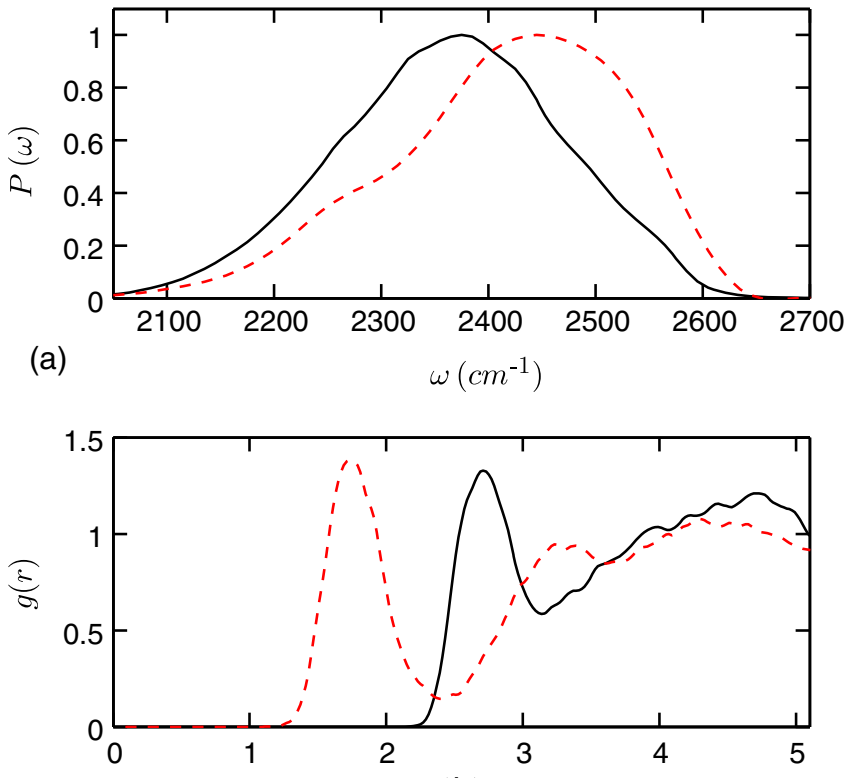

(b)

$\mathrm{r}(\stackrel{\circ}{\circ})$

Figure 1. (a) The distribution of OD stretch frequencies of the bulk modes (solid) and hydration shell OD bonds (dashed), each normalized to the maximum value of 1 . (b) The radial distribution functions between acetone and water molecules. The solid and dashed curves are for acetone oxygen $\left(\mathrm{O}_{a}\right)$-water oxygen $(\mathrm{O})$ and acetone oxygen $\left(\mathrm{O}_{a}\right)$-water deuterium (D) correlations.

(a)
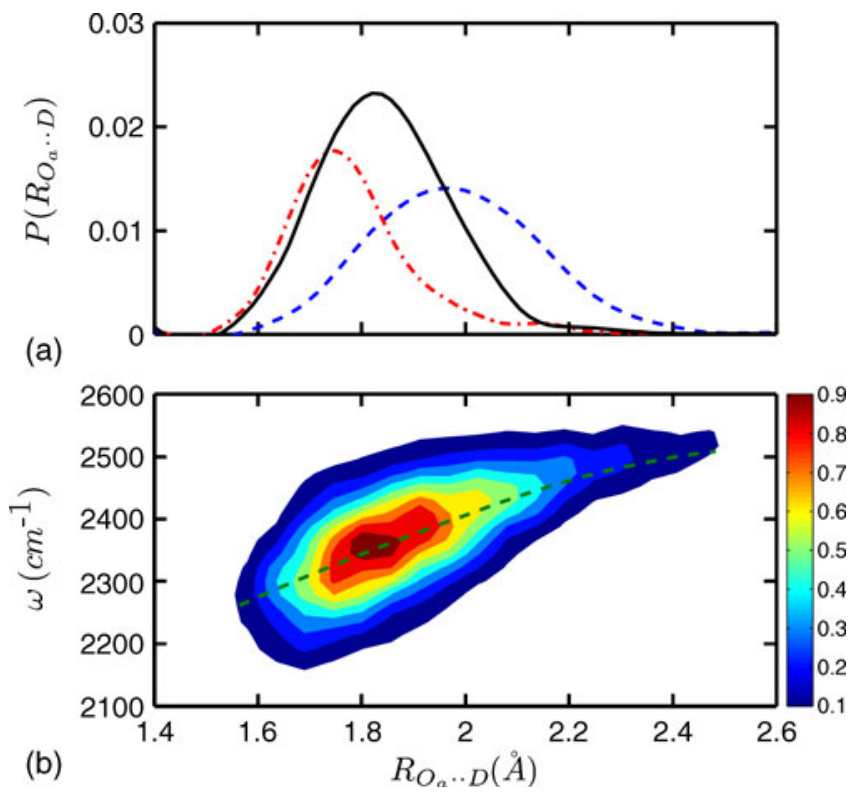

Figure 2. (a) The distribution of the $O_{a} \cdot \mathrm{D}$ distance for fixed values of the OD frequency. The solid, dashed-dotted and dashed curves are for OD frequency $\Delta \omega=0 \pm 5 \mathrm{~cm}^{-1}$, $-100 \pm 5 \mathrm{~cm}^{-1}$ and $100 \pm 5 \mathrm{~cm}^{-1}$, respectively, where $\Delta \omega$ represents the deviation from the average frequency. (b) Joint probability distribution of OD frequency and $O_{a} \cdot$ D distance. The contour levels of different fractions of the maximum value are shown in different colour codes. The results are for water molecules in the acetone hydration shell. 
OD groups are found to be 2414 and $2368 \mathrm{~cm}^{-1}$, respectively. The present value of the average frequency of bulk OD groups is very close to the corresponding result for pure water $\left(2380 \mathrm{~cm}^{-1}\right),{ }^{34}$ the small difference may be attributed to the influence of the acetone solute on water molecules beyond the first hydration shell. The present result of the blue shift in the stretch frequency of hydrated OD bonds is in general agreement with experimental findings of infrared spectroscopy results for a mixture with low acetone content in water. ${ }^{10}$

Next, we perform further analysis of the relation between the frequency of an OD bond in the hydration shell and the $\mathrm{D} \cdot \mathrm{O}_{a}$ hydrogen bond distance. The results are now averaged over all the OD groups pointing toward the $\mathrm{O}_{a}$ in its hydration shell. In figure $2 \mathrm{a}$, we have shown the distribution of $\mathrm{D} \cdot \mathrm{O}_{a}$ distance for three fixed values of the OD frequency (within $\pm 5 \mathrm{~cm}^{-1}$ ). It is seen that as the frequency increases, the corresponding distribution of $\mathrm{D} \cdot \cdot \mathrm{O}_{a}$ distance is also shifted toward its larger values. Although the distributions are fairly wide with significant overlaps, a one-to-one frequencystructure correlation can be extracted on average. This is more clearly shown in figure $2 b$ where we have shown the contour plots of the conditional probability distributions of observing a particular frequency for a given $\mathrm{D} \cdot \mathrm{O}_{a}$ distance. Clearly, there is a substantial width in the probability distributions which rules out the possibility of assigning a single instantaneous frequency to a given $\mathrm{D} \cdot . \mathrm{O}_{a}$ distance. On an average, however, the frequency is seen to be a monotonic function of the $\mathrm{D} \cdot \cdot \mathrm{O}_{a}$ distance, as shown by the dashed line in figure $2 b$, which means, like pure water, ${ }^{39,63-66} a$ frequency-structure correlation is also present on average in the acetone hydration shell where the frequency of an OD bond decreases with decrease of the associated $\mathrm{D} \cdot \mathrm{O}_{a}$ hydrogen bond distance. We note that a first principles calculation of such conditional probability distributions for hydration shells of acetone solute is presented here for the first time. Our calculations of the power spectra of the velocity time correlation of the $\mathrm{D}$ atoms of hydration shell water molecules (figure not shown) also show a blue shift of the stretch band for the hydration shell water when compared with the corresponding bands of bulk water. Such shifts of the power spectral bands for hydration shell water molecules are consistent with the results of figure 1 and also with experimental results of infrared studies. ${ }^{10}$

\section{Dynamics of acetone-water hydrogen bonds and escape of water from hydration shell of acetone}

We have used the so-called population correlation function approach $^{39,67-77}$ to investigate the dynamics of acetone-water hydrogen bonds. To study the breaking dynamics of acetone-water hydrogen bonds, we calculate the so-called continuous hydrogen bond time correlation function which describes the probability that an initially hydrogen bonded acetone-water pair remains bonded at all times up to $t .{ }^{39,68-70,72,76,77}$ The associated integrated relaxation time $\tau_{H B}$ gives the average lifetime of a hydrogen bond between acetone and a water molecule in its hydration shell. Integration of $S_{H B}(t)$ yields a value of $1.36 \mathrm{ps}$ for $\tau_{H B}$. This acetone-water hydrogen bond lifetime is slightly shorter than the lifetimes of hydrogen bonds in pure water which was found to be $\sim 2$ ps. ${ }^{39}$ We note in this context that the time scale of acetone-water hydrogen bond relaxation was found to be longer in reference 16 due to confinement effects. In the system of reference 16 water was surrounded by acetone at high concentration which is very different from the system of present study. We next calculated the residence times of water molecules in the acetone hydration shell by following a similar population correlation function approach. ${ }^{76,77}$ In particular, we calculated the residence correlation function $S_{R}\left(t ; t^{*}\right)$ which describes the probability that a water molecule, which was in the hydration shell of the acetone molecule at time $t=0$, remains continuously in the same hydration shell up to time $t$ subject to the allowance time $t^{*}$. The associated integrated relaxation time, $\tau_{R}$, gives the average residence time of a water molecule in the molecule hydration shell. We calculated the residence time, $\tau_{R}$, by explicit integration of $S_{R}\left(t ; t^{*}\right)$ from simulations until $5 \mathrm{ps}$ and by calculating the integral for the tail part from fitted exponential functions. Following previous work, ${ }^{76-78}$ we took the allowance time to be 2 ps for the continuous residence function and found a value of $11.7 \mathrm{ps}$ for the residence time of water molecules in the acetone solvation shell.

\section{Vibrational spectral diffusion of hydration shell water: Frequency time correlation function}

In this section, we calculate the time correlations of fluctuating stretch frequencies of water molecules that are found in the acetone hydration shell at the initial time. We note that this frequency time correlation function serves as a key dynamical quantity in the studies of vibrational spectral diffusion. This correlation function is defined as

$$
C_{\omega}(t)=<\delta \omega(t) \delta \omega(0)>/<\delta \omega(0)^{2}>,
$$

where $\delta \omega(t)$ is the fluctuation from the average frequency at time $t$. The average of equation 1 is over the initial time and over all the OD groups present 
initially in the hydration shell of acetone. The results of the frequency time correlation are shown in figure 3.

We observe a fast decay and a weak oscillation at short times followed by slower decay extending to a few ps. As before, ${ }^{39,66}$ we used the following function including a damped oscillatory function to fit the calculated results of spectral diffusion

$$
\begin{gathered}
f(t)=a_{0} \cos \omega_{s} t e^{-t / \tau_{0}}+a_{1} e^{-t / \tau_{1}} \\
+\left(1-a_{0}-a_{1}\right) e^{-t / \tau_{2}}
\end{gathered}
$$

and found time scales of $0.080,1.5$ and 12 ps with weights of $0.56,0.33$ and 0.11 , respectively. The frequency of the damped oscillation is found to be about $100 \mathrm{~cm}^{-1}$. We carried out a separate calculation of the power spectra of the relative velocity of initially hydrogen bonded $\mathrm{O} \cdot \cdot \mathrm{O}_{a}$ pairs (results not shown). Enhanced intensities are found at around 40 and $115 \mathrm{~cm}^{-1}$ in the power spectra arising from intermolecular bending and stretching vibrations of the hydrogen bonded acetonewater pairs. Both bending and stretching modes of intermolecular vibrations can modulate the OD frequencies and hence contribute to the short-time oscillation of the spectral diffusion. It is also clear from the results of the previous section that the two slower relaxation times of spectral diffusion correspond to the lifetimes of acetone-water hydrogen bonds and the residence time of water in the acetone hydration shell, respectively. In a dynamical equilibrium situation, hydrogen bonds continuously break and reform and also water molecules leave and enter the acetone hydration shell and these dynamical processes alter the OD frequencies and induce characteristic time scales in the decay of the spectral diffusion of hydration shell water molecules.

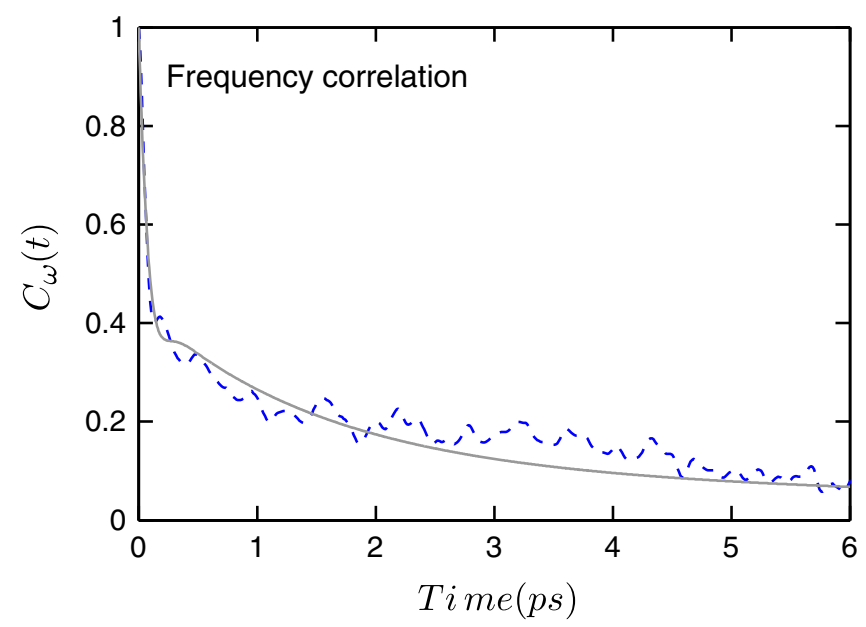

Figure 3. The time correlation functions of OD fluctuating frequencies averaged over water molecules of solvation shell of acetone oxygen at the initial time. The grey solid curve represents the fit by the function as given by equation 2 .
We note that the presence of three time scales as discussed above is found when vibrational spectral diffusion calculations are done exclusively for those water molecules which were in the hydration shell of the carbonyl oxygen of the acetone molecule. When the spectral diffusion calculations are done over all the OD modes of the solution, only two time scales corresponding to intermolecular hydrogen bond stretch and hydrogen bond lifetime are found without the third time scale of the residence time. This is likely due to the rather small weight that hydration shell OD groups make to the overall spectral diffusion in the presence of only one acetone molecule in the solution.

\section{Conclusions}

We have presented a theoretical study of the hydrogen bond dynamics and vibrational spectral diffusion in aqueous solution of an acetone molecule at room temperature. Our calculations are based on ab initio molecular dynamics for trajectory generation and wavelet analysis for frequency calculations without involving any empirical potential parameters. We first looked at the frequency distributions and frequency-structure correlations of water molecules in the acetone hydration shell and found that the frequencies of OD bonds in the hydration shell, i.e., those which are hydrogen bonded to the carbonyl oxygen of acetone, have higher stretch frequencies than those in the bulk water in agreement with experimental observations. ${ }^{10}$ Also, on average, the frequencies of hydration shell OD modes are found to increase with increase in the acetone-water hydrogen bond distance, thus establishing the presence of a frequency-distance correlation which is so crucial in the interpretation of vibrational spectral diffusion experiments.

On the dynamical side, we found that the acetonewater hydrogen bonds have a shorter lifetime than water-water hydrogen bonds. When the vibrational spectral diffusion is studied exclusively for water in the hydration shell of the carbonyl oxygen, the dynamics revealed three time scales: a short-time relaxation of $\sim 80 \mathrm{fs}$ corresponding to the dynamics of intact acetone-water hydrogen bonds, a slower relaxation of $\sim 1.3$ ps corresponding to the acetone-water hydrogen bond lifetime and another longer time constant $\sim 12 \mathrm{ps}$ corresponding to the escape dynamics of water from the acetone hydration shell. However, the longest time scale was not noticed when the spectral diffusion was calculated by averaging the over all water molecules which is likely due to the rather small weight contribution that the hydration shell water makes to the overall behaviour for a single solute. 
In the present study, we have considered only one acetone molecule dissolved in water. It would be interesting to consider such solutions at higher acetone concentrations and investigate possible non-ideal aspects in equilibrium and dynamical properties. In particular, concentrated water-acetone solutions have been reported to show aggrerate formation when the size of the simulations systems are big enough. ${ }^{13}$ In the present case, we have considered a rather small system because of the use of ab initio molecular dynamics methodology. Although, the goal of the present study has been to look at the behaviour of water molecules in hydration shells of acetone from spectral diffusion point of view, it would nevertheless be worthwhile to consider bigger systems in future studies to explore such aggregate formation kinetics from first principles. It would also be interesting to investigate aqueous solutions of other molecular solutes such as alcohols, amino acids and peptides. We hope to address some of these systems in our future work.

\section{Acknowledgements}

The authors thank the Department of Science and Technology (DST), Government of India for financial support to carry out this work.

\section{References}

1. Löwen B and Schulz S 1995 Thermochim. Acta 26269

2. Matteoli E and Lepori L 1984 J. Chem. Phys. 802856

3. Blandamer M J, Blundell N J, Burgess J, Cowles H J and Horn I M 1990 J. Chem. Soc., Faraday Trans. 86283

4. Blandamer M J, Burgess J, Cooney A, Cowles H J, Horn I M, Martin K J, Morcom K W and Warrick P Jr $1990 \mathrm{~J}$. Chem. Soc., Faraday Trans. 862209

5. Marcus Y 2001 Monatsh. Chem. 1321387

6. Landoldt-Bönstein 1977 Numerical data and functional relationships in science and technology (New Series, Group IV, Vol. 1, Springer-Verlag, New York)

7. McCall D W and Douglass D C 1967 J. Phys. Chem. 71 987

8. Goldammer E T and Hertz H G 1970 J. Phys. Chem. 74 3734

9. Venables D S and Schmuttenmaer C A 2000 J. Chem. Phys. 11311222

10. (a) Max J J and Chapados C 2003 J. Chem. Phys. 119 5632; (b) 2004 J. Chem. Phys. 1206625

11. Ferrario M, Haughney M, McDonald I R and Klein M L 1990 J. Chem. Phys. 935156

12. Freitas L C G, Cordeiro J M M and Garbujo F L L 1999 J. Mol. Liq. 791

13. Perera A and Sokolic F 2004 J. Chem. Phys. 12111272

14. Weerasinghe S and Smith P E 2003 J. Chem. Phys. 118 10663

15. Liang W, Li H, Lei Y and Han S 2004 J. Mol. Struct. 686109
16. Gillijamse J J, Lock A J and Bakker H J 2005 Proc. Natl. Acad. Sci. USA 1023202

17. Gupta R and Chandra A 2007 J. Chem. Phys. 127 024503

18. Bernasconi L and Sprik M 2003 J. Chem. Phys. 119 12417

19. Neugebauer J, Louwerse M J, Baerends E J and Wesolowski T A 2005 J. Chem. Phys. 122094115

20. Crescenzi O, Pavone M, Angeles F and Barone V 2005 J. Phys. Chem. B 109445

21. Pavone M, Crescenzi O, Morelli G, Rega N and Barone V 2006 Theor. Chem. Acc. 116456

22. Thompson M 1996 J. Phys. Chem. 10014492

23. Rohrig U F, Frank I, Hutter J, Laio A, VandeVondele J and Rothlisberger U 2003 Chem. Phys. Chem 41177

24. Aidas K, Kongsted J, Osted A, Mikkelsen K and Christiansen O 2005 J. Phys. Chem. A 1098001

25. Car R and Parrinello M 1985 Phys. Rev. Lett. 552471

26. Marx D and Hutter J 2000 in Modern methods and algorithms of quantum chemistry (ed) J Grotendorst (NIC, FZ Jülich, ADDRESS) for downloads see $<$ www. theochem.ruhr-uni-bochum.de/go/cprev.html>

27. Fuentes M, Guttorp P and Sampson P D 2007 in Statistical methods for spatio-temporal systems, chapter 3 (eds) B Finkenstadt, L Held and V IshamChapman (Boca Raton: Hall/CRC)

28. Vela-Arevalo L V and Wiggins S 2001 Int. J. Bifur. Chaos 111359

29. Semparithi A and Keshavamurthy S 2003 Phys. Chem. Chem. Phys. 55051

30. Hutter J, Alavi A, Deutsch T, Bernasconi M, Goedecker $\mathrm{S}$, Marx D, Tuckerman $\mathrm{M}$ and Parrinello M CPMD program, mPI für Festkörperforschung and IBM Zurich Research Laboratory

31. Troullier N and Martins J L 1991 Phys. Rev. B 431993

32. (a) Becke A D 1988 Phys. Rev. A 38 3098; (b) Lee C, Yang W and Parr R G 1988 Phys. Rev. B 37785

33. Laasonen K, Sprik M, Parrinello M and Car R 1993 J. Chem. Phys. 999080

34. (a) Silvestrelli P L and M. Parrinello 1999 Phys. Rev. Lett. 82 3308; (b) 1999 J. Chem. Phys. 111 3572; (c) Silvestrelli P L, Bernasconi M and Parrinello M 1997 Chem. Phys. Lett. 277478

35. (a) Krack M, Gambirasio A and Parrinello M $2002 \mathrm{~J}$. Chem. Phys. 117 9409; (b) Chen B, Ivanov I, Klein M L and Parrinello M 2003 Phys. Rev. Lett. 91215503

36. Izvekov S and Voth G 2002 J. Chem. Phys. 11610372

37. Sprik M, Hutter J and Parrinello M 1995 J. Chem. Phys. 1051142

38. VandeVondele J, Mohamed F, Krack M, Hutter J, Sprik M and Parrinello M 2005 J. Chem. Phys. 122014515

39. (a) Mallik B S, Semparithi A and Chandra A 2008 J. Phys. Chem. A 112 5104; (b) Mallik B S and Chandra A 2008 J. Mol. Liq. 14331

40. (a) Boero M, Terakura K, Ikeshoji T, Liew C C and Parrinello M 2000 Phys. Rev. Lett. 85 3245; (b) 2001 J. Chem. Phys. 115 2219; (c) Boero M 2007 J. Phys. Chem. A 11112248

41. Mallik B S and Chandra A 2008 J. Phys. Chem. A 112 13518

42. (a) Marx D, Tuckerman M E, Hutter J and Parrinello M 1999 Nature 397 601; (b) Tuckerman M E, Marx D and Parrinello M 2002 Nature 417925 
43. Kirchner B, Stubbs J and Marx D 2002 Phys. Rev. Lett. 89215901

44. Heuft J M and Meijer E J 2006 Phys. Chem. Chem. Phys. 83116

45. Cavallaria M, Cavazzoni C and Ferrario M $2004 \mathrm{Mol}$. Phys. 102959

46. Ikeda T, Hirata M and Kimura T 2003 J. Chem. Phys. 11912386

47. Leung K and Rempe S B 2004 J. Am. Chem. Soc. 126 344

48. (a) Ramaniah L M, Barnasconi M and Parrinello M 1999 J. Chem. Phys. 111 1587; (b) Lyubartsev A P, Laaksonen $\mathrm{K}$ and Laaksonen A 2001 J. Chem. Phys. 1143120

49. Gaigeot M P and Sprik M 2004 J. Phys. Chem. B 108 7458

50. Mallik B S, Semparithi A and Chandra A 2008 J. Chem. Phys. 129194512

51. Tsuchida E, Kanada Y and Tsukada M 1999 Chem. Phys. Lett. 311236

52. Paglial M, Cardini G, Righini R and Schettino V 2003 J. Chem. Phys. 1196655

53. Morrone J A and Tuckerman M E 2002 J. Chem. Phys. 1174403

54. Diraison M, Martyna G J and Tuckerman M E $1999 \mathrm{~J}$. Chem. Phys. 1111096

55. Boese D, Chandra A, Martin J M L and Marx D $2003 \mathrm{~J}$. Chem. Phys. 1195965

56. Morrone J A, Haslinger K E and Tuckerman M E 2006 J. Phys. Chem. B 1103712

57. (a) Boero $\mathrm{M}$, Ikeshoji $\mathrm{T}$, Liew $\mathrm{C} \mathrm{C}$, Terakura $\mathrm{K}$ and Parrinello M 2004 J. Am. Chem. Soc. 126 6280; (b) Mundy C J, Hutter J and Parrinello M $2000 \mathrm{~J}$. Am. Chem. Soc. 1224837

58. (a) Tuckerman M, Chandra A and Marx D 2006 Acc. Chem. Res. 39 151; (b) Chandra A, Tuckerman M and Marx D 2007 Phys. Rev. Lett. 99145901

59. Marx D, Chandra A, Tuckerman M 2010 Chem. Rev. 110 2174
60. Berendsen H J C, Grigera J R and Straatsma T P 1987 J. Phys. Chem. 916269

61. Jorgensen W L, Maxwell D and Rives J T 1996 J. Am. Chem. Soc. 11811225

62. Matlab, version-R2007a, The MathWorks, Inc., USA (http://www.mathworks.com)

63. (a) Lawrence C P and Skinner J L 2003 J. Chem. Phys. 118 264; (b) Chem. Phys. Lett. 3694722003

64. Corcelli S A, Lawrence C P and Skinner J L 2004 J. Chem. Phys. 1208107

65. Rey R, Moller K B and Hynes J T 2002 J. Phys. Chem. A 10611993

66. Moller K B, Rey R, Hynes J T 2004 J. Phys. Chem. A 1081275

67. Lee H S and Tuckerman M E 2007 J. Chem. Phys. 126 164501

68. Rapaport D 1983 Mol. Phys. 501151

69. (a) Chandra A 2000 Phys. Rev. Lett. 85 768; (b) Chowdhuri S and Chandra A 2002 Phys. Rev. E 66 041203; (c) Paul S and Chandra A 2004 Chem. Phys. Lett. 386218

70. Balasubramanian S, Pal S and Bagchi B 2002 Phys. Rev. Lett. 89115505

71. Luzar A and Chandler D 1996 Nature 37953

72. Luzar A 2000 J. Chem. Phys. 11310663

73. Xu H and Berne B J 2001 J. Phys. Chem. B 105 11929

74. Xu H, Stern H A and Berne B J 2002 J. Phys. Chem. B 1062054

75. Schreiner E, Nicolini C, Ludolph B, Ravindra R, Otte N, Kohlmeyer A, Rousseau R, Winter R and Marx D 2004 Phys. Rev. Lett. 92148101

76. (a) Chowdhuri S and Chandra A 2006 J. Phys. Chem. B 110 9674; (b) Chandra A and Chowdhuri S 2002 J. Phys. Chem. B 1066779

77. Chandra A 2003 J. Phys. Chem. B 1073899

78. Impey R W, Madden P A, McDonald I R 1983 J. Phys. Chem. 875071 